Southern Illinois University Edwardsville SPARK

SIUE Faculty Research, Scholarship, and Creative Activity

$11-27-2015$

\title{
Climate Change and Interpersonal Violence: A "Global" Estimate and Regional Inequities
}

Dennis Mares

Southern Illinois University Edwardsville, dmares@siue.edu

Kenneth W. Moffett

Southern Illinois University Edwardsville, kmoffet@siue.edu

Follow this and additional works at: http://spark.siue.edu/siue_fac

Part of the Criminology Commons, Other Environmental Sciences Commons, and the Other Political Science Commons

\section{Recommended Citation}

Mares, Dennis and Kenneth W. Moffett Forthcoming.Climate Change and Interpersonal Violence: A "Global" Estimate and Regional Inequities. Climatic Change.

This Article is brought to you for free and open access by SPARK. It has been accepted for inclusion in SIUE Faculty Research, Scholarship, and Creative Activity by an authorized administrator of SPARK. For more information, please contact gpark@siue.edu. 


\section{Cover Page Footnote}

This is an Accepted Manuscript of an article published by Springer in Climatic Change on November 27, 2015, available online:

http://dx.doi.org/10.1007/s10584-015-1566-0 


\title{
Climate Change and Interpersonal Violence: A "Global" Estimate and Regional Inequities
}

\author{
Authors: Dennis M. Mares ${ }^{1 *}$, Kenneth W. Moffett ${ }^{2}$
}

\begin{abstract}
Affiliations:
${ }^{1}$ Southern Illinois University Edwardsville. Department of Sociology and Criminal Justice Studies

${ }^{2}$ Southern Illinois University Edwardsville. Department of Political Science

*Correspondence to: dmares@ @iue.edu, Peck Hall, Box 1455 Edwardsville, IL 62026, 618-2137045
\end{abstract}

Total $=6448$ words

\begin{abstract}
This study estimates the predicted impact of climate change on levels of violence in a sample of 57 countries. We sample western and non-western countries and perform a multilevel ARFIMA regression to examine if warmer temperatures are associated with higher levels of homicide. Our results indicate that each degree Celsius increase in annual temperatures is associated with a nearly $6 \%$ average increase in homicides. Regional variation in this predicted effect is detected, for example, with no apparent effects in former Soviet countries and far stronger effects found in Africa. This variation indicates that climate change may acutely increase violence in areas that already are affected by higher levels of homicides and other social dislocations.
\end{abstract}

Key Words: Violence, Homicide, Climate Change, International 


\section{Introduction ${ }^{1}$}

Although an increasing body of work examines the link between climate change and intergroup conflict, the connection between climate change and interpersonal violence has received scant attention from scholars (Abbott 2008; Barnett 2003; Hendrix and Glaser 2007; Buhaug, Gleditsch and Theisen 2008; Tol and Wagner 2010; Landis 2014; Hsiang and Burke 2014; Hsiang, Burke and Miguel 2013). Existing studies, however, generally find significant positive relationships between interpersonal violence and climate change (Landis 2014; Rotton and Cohn 2003; Anderson, Bushman and Groom 1997; Ranson 2014; Mares 2013a; Mares 2013b). Unfortunately, many of the studies that examine interpersonal violence are limited to Western countries, especially the United States (c.f., Hsiang, Burke and Miguel 2013; Blakeslee and Fishman 2014; Ikegaya, and Suganami 2008). Moreover, violence and climate change are often measured in radically different manners making it difficult to draw comparisons between studies and estimate how climate change may affect levels of interpersonal violence worldwide. Our study seeks to a assuage some of this criticism. First, we use a broad sample of countries and a stable cross-national measure of violence to examine the likely 'global' impact of climate change on violence. Second, our study explores if climatic effects are context-dependent. Mares (2013a), for instance, found that temperature increases play no apparent role in levels of violence in affluent neighborhoods, but in economically disadvantaged neighborhoods a strong positive association between rising temperatures and violence was detected. In this study, we examine if such effects may also exists across countries.

We examine 57 countries, and discover a significant positive relationship between climate change and homicides, even after controlling for a variety of socio-economic factors. Our overall model suggests that 'globally' for each degree Celsius increase in annual temperatures in a typical country, homicides on average are predicted to increase by about $5.9 \%$. Once countries are separated by region, however, results diverge. We note particularly strong effects in Africa, but did not find significant results for Europe, Asia and former Soviet countries. Our results support prior research about the link between climate change and violence, however, we also find that climate change likely does not affect levels of violence equally across countries.

\section{Review of Previous Research}

The link between warmer temperatures and interpersonal violence has been studied since the 19th century (Quetelet 1842). Although theoretical differences about the causal mechanisms between heat and violence persist, many studies find support for the two most commonly measured explanatory models, Routine Activities Theory (RAT) and General Affective Aggression Model (GAAM) (Felson and Cohen 1979; Anderson et al. 2000). RAT theory explains that increasing temperatures likely increases the frequency of interactions between people, thereby raising the number of violent encounters (Baumer and Wright 1996; Breetzke and Cohn 2011; Ceccato 2005; Cohn and Rotton 1997, 2000, 2003, 2005; Hipp, Bauer and Curran 2004 Lebeau 1990; Lebeau and Corcoran 1994; Lebeau and Langworthy 1986; Farrell and Pease 1994; Rotton and Cohn 2000; 2003). This theory suggests, for instance, that during pleasant temperatures people exit their homes and go to bars or restaurants where they are more likely to encounter others. All things being equal, increased encounters should increase violent interactions. 
GAAM builds on previous heat-aggression theories and explains increases in violence as a result of heightened aggressive emotions brought on by warmer conditions (Anderson 1989; Anderson and Anderson 1998; Anderson, Anderson, Dorr and DeNeve 2000; Baron 1972; Baron and Bell 1976; Bell and Baron 1976; Kenrick and MacFarlane 1986; Reifman, Larrick and Fein 1991). In this theory, heat raises irritability levels, which leads to increased aggressiveness.

Research has found overwhelming evidence for a positive relationship between violence and temperature. Most of this prior research, however, examines 'seasonality effects', specifically focusing on how violence varies over months, weeks, or even hours (Hipp, Bauer and Curran 2004). Therefore, many of these studies really focus on 'weather' and few have specifically studied the link between climate change and violence. Nonetheless, the few studies that focus on climate change also generally report strong associations between rising temperatures and violence (Cohn and Rotton 2003; Anderson and DeLisi 2011, Mares 2013b; Ranson 2014).

The causal links between climate change and violence may be more complex than examining temperature and violence levels over time. Climate change may increase violence in multiple indirect ways. Anderson and DeLisi (2011) suggest that beyond direct heat effects, growing malnutrition may prime individuals for higher levels of aggression. Furthermore, they suggest that higher levels of 'eco' migration and growing social conflict may contribute to increases in homicides. Agnew (2011) likewise fingers higher than usual temperatures and food shortages in raising crime levels, but also suggests that growing inequality and poverty triggered by climate induced economic instability may contribute to an increase in violence.

There may be many ways in which climate change can affect violence. Empirically speaking, it is wise to clarify which effects are most likely and find some support in recent research on violence. To wit, many studies question the link between migration and crime with many recent studies finding significant negative relationships (Martinez 2014). It also remains unclear whether natural disasters, such as hurricanes push up crime rates (Zahran et al. 2009; Varano et al. 2010). Social conflict may increase as a result of climate change (Hsiang, Burke, Miguel 2011, 2013), but that does not necessarily mean that growing social conflict increases interpersonal violence. While some have linked decreases in state 'legitimacy' (LaFree and Tseloni 2006) to increases in violence, there generally appears little support for brutalization effects related to wars (Mares 2009). Finally, a long line of research has linked various, but not all, aspects of economic growth (or decline) to increases in violence (Messner and Rosenfeld 1997; LaFree and Tseloni 2006).

Given the support for the link between temperature and violence, it stands to reason that this will be the primary way in which climate change will affect violence, at least in the foreseeable future. This is not say that other more indirect elements play no role, or that the relative contribution of other factors may not change as climate change becomes more pronounced and its social effects spread more broadly. Prior research on weather, climate change and violence leads one to expect a similar positive association between indicators of climate change (temperature in particular) and violence. Any indirect effects of climate change (migration, health, economy, etc.) on violence are likely to be highly context dependent and at present, appear somewhat contested in the predicted effect they may have overall levels of violence.

Measuring the actual link between climate change and violence is more complex than theorizing about the possible relationships. Studies explicitly examining climate change and 
violence (or crime more broadly) incorporate climatological measures as proxies for climate change (Cohn and Rotton 2003, Anderson \& DeLisi 2011; Hsiang, Burke and Miguel 2013; Mares 2013a; 2013b; Ranson 2014), but do not incorporate more indirect ways in which climate change may affect violence. We suspect that the latter will need to be done in smaller scale settings, as it is impractical for the current wave of macro-level studies, including our own, to model the many and interactive factors associated with climate change.

We do expect that the positive association between climate change and violence will generally hold in a cross-national study. In the seasonality and violence context, however, several studies found that excessive heat can actually reduce violence as people seek shelter from extreme heat (Cohn and Rotton 2005). Nonetheless, this effect is criticized in the literature as well (Bushman, Wang, Anderson 2005a, 2005b). In the context of climate change, such 'curvilinear' effects may need to be reexamined as climate change is predicted to increase the number of extreme heat events (Archer and Rahmstorf 2010; Karl Melillo and Peterson 2009). In a cross-national study, however, such effects are unlikely to appear as the resolution of climate and violence data is insufficient.

While issues surrounding measurement and curvilinear effects cannot be easily resolved given current available data, there are two problems in the climate change literature that can be addressed. One issue is the spatial bias of current research, which has primarily examined data from the United States. Even the most comprehensive meta-analysis by Hsiang, Burke and Miguel (2013) is affected by this spatial bias as the majority of the studies in their analysis are from the United States or Europe. We suspect that certain types of homicides, particularly those more likely to occur outdoors are more susceptible to warming temperatures.

A related issue that emerges from current research is whether climate change affects violence equally across space. Several studies examining seasonality factors indicate that crime and violence fluctuate with a higher seasonal amplitude in more economically depressed communities (Ceccato 2005). In the context of climate change, Mares (2013a) finds that economically disadvantaged neighborhoods experience a stronger and more significant association between rising temperatures and violence. It is unknown, however, if such differential impacts can be found at higher levels of aggregation.

In the following sections, we address two aspects of the climate change-violence relationships that have not yet been adequately explored. First, we sample a large number of countries and estimate the predicted country-level impact of climate change on homicides. In doing so, we develop a more refined estimate of the link between violence and climate change. Because prior research indicates that different places respond differently to climatic effects, we also examine if there is any variation in this relationship across regions. Based on prior research, we expect to find a generally positive association between our indicators of climate change and violence. However, we also find it likely that the effects of climate change may vary substantially across groups of countries.

\section{Data and Methodology}

The current study relies on several data sources in order to make a more accurate prediction of the likely link between climate change and violence. ${ }^{2}$ Different types of offenses may respond uniquely to climatic shifts. Previous studies that use a full range of violent offenses (typically, 
homicide, rape, assault and robbery) find strongest results for assaults and sometimes find nonsignificant results for homicides (Rotton and Cohn 2003; Ranson 2014; Mares 2013b). Assaults and robberies are a good indicator of trends of violence within one country, but using them as an indicator for violence is problematic in an international context. Both are often underreported, and definitions of assaults vary across legal boundaries reducing the consistency of the measure.

Homicide is less beset by definitional problems and is generally regarded as a stable indicator of trends in violence across space and time (Rosenfeld and Messner 1997; Mares 2009; Ouimet and Montmagny-Grenier 2014; Liem et al. 2013). For cross-national research, homicide is the most valid indicator of violence because it is most likely to be consistently reported, detected and defined. Because homicides correlate very well with trends in other violent crimes they are often used as a predictor for violent crime trends in general (Rosenfeld 2000). This means that a positive relationship between climate change and homicide likely indicates a positive relationship between climate change and violence in general. For this study, therefore, homicides data from the United Nations (UN) are utilized. In most cases, those data are collected from law enforcement sources, thus representing criminal homicides (excluding defensive use of force and police killings). The data are limited in time, covering a relatively short time period between 1995 and 2012. For some countries missing data reduce this time frame even further. The database on countries is comprised of data for 57 countries that have enough annual homicides on which to conduct our analysis. ${ }^{3}$ More information about the way in which the data were gathered and analyzed is available in the Appendix and the supplementary materials.

Temperature data were collected from the Global Historical Climatology Network (GHCN) operated by the National Oceanographic and Atmospheric Administration (NOAA). Rather than using national estimates for average annual temperatures, we use annual temperatures from one or several populous cities for each country. This approach mirrors that taken by Rotton and Cohn (2003) and Anderson and DeLisi (2011). Given that violent crimes are typically heavily concentrated in urban places, it would not be correct to use nationally averaged temperature data. Temperature data from large urban centers, in which homicides occur in greater concentrations, are therefore more relevant to examine the linkages between temperature increases and violence. ${ }^{4}$ Ideally, we would explore the relationship between city homicide and city climate data, but the lack of urban specific homicide data severely restricts our sample size.

One may suggest that our timeframe is too short to measure climate change, and to some extent that is correct. Our goal, however, is to explore the potential link between climate change and violence, not to show the existence of climate change. While it is impossible to fully attribute any specific yearly temperature changes to either climate change or typical natural variation, we are confident that the number of years in combination with the large number of countries should be sufficient to explore the likely nature of the relationship. Hsiang, Burke and Miguel (2013) received criticism because many of the studies in their meta-analysis on climate change and violence use temperature data as a proxy for climate change. Two of the authors (Hsiang and Burke 2014) recently defended the use of such data. We concur with those authors that the experience of climate change is a continuous event. In addition, offenders likely do not care if the weather conditions they experience prior to offending are (in part) the result of climate change or normal variation; it is relatively warm, or it is not. By coupling the experience of specific climatic conditions to offending patterns we can extrapolate - if we know the overall contribution of climate change during a specific time period - how much of this offending is likely attributable to climate change (Dell, Jones and Olken 2014). Although temperature data 
are sometimes available at the monthly level, we were unable to capitalize on such data for this study as most countries only report annual homicide data. We do not feel this is a major setback, as studies using either monthly or annual data have yielded similar results (Mares 2013a, 2013b; Ranson 2014; Cohn and Rotton 2003, Anderson and DeLisi 2011).

Our study controls for several effects that are known to likely alter homicide levels. ${ }^{5}$ World Bank data were examined to provide contextual information, including population, social, economic and health indicators. Population data were used to create country specific homicide rates, which were subsequently log transformed to normalize their distribution.

Control factors included in our final models are the consumer price index, male youth unemployment, infant mortality (per 1,000), immigrant population (percent of total), and casualties of war (per 100,000). While we would have preferred to include additional measures beyond those explored here, doing so would have meant excluding more sites from our study, or allow multicollinearity to influence our results. ${ }^{6}$ Recent work on climate change also cautions against including too many control variables, particularly if there is concern that these controls themselves are influenced by climate change (Dell, Jones and Olken 2014).

The countries included in the study are not a random sample, but rather a convenience sample of available data. ${ }^{7}$ Developed countries typically have better recording practices, meaning their data are more easily available then that of developing countries. The results of our analysis are therefore not fully representative of all countries. Our current sample is somewhat biased toward developed countries, which is fairly typical for cross-national studies of violence (Liem et al. 2013; Mares 2009). Of 57 countries, six are from Africa, thirteen from Latin America, and ten from Asia. While not global in the true meaning of the word, our current sample incorporates a diverse group of countries that comprise a substantial portion of the total global population (over 3 billion). What is more, the countries in the sample display temperature increases in line with expected global land surface temperature anomalies (see Figure 1). We therefore believe our estimate reveals the typical effects that countries are likely to experience.

-Figure 1 about here-

\subsection{Methodology and Estimation Technique}

We have a repeated cross-sectional (RCS) dataset, since our dataset consists of 57 countries over seventeen years. To test our theoretical expectations, we utilize a multilevel, ARFIMA regression with varying slopes and intercepts. Multilevel models (MLMs) allow us to model homicide rates at the country and time levels simultaneously. Had we utilized a traditional fixedeffects model in place of the one reported here, we would only explain variance in homicide rates across countries while accounting for the time-series nature of the data. ${ }^{8}$ However, the data also contain variation in homicide rates within countries. The traditional fixed-effects model forces us to exclude an important source of variance in our dependent variable: homicide rates within countries (see Gelman and Hill 2007; Lebo and Weber 2015). Since our goal is to explain variance in homicide rates while fully leveraging the RCS dataset, MLMs are a better approach.

A multilevel model with varying slopes and intercepts fully capitalizes on the structure of this dataset, since the systematic variance in homicide rates comes from two primary sources: countries and time. A multilevel model also allows us to capitalize on the RCS dataset, where 
heterogeneity exists between and within time-points (Lebo and Weber 2015, 244). If we solely relied upon multilevel modeling with an OLS regression, though, we would not sufficiently account for the time-series component of our dataset (Lebo and Weber 2015). ${ }^{9}$ More specifically, we would not adequately consider the problems presented by autocorrelation, nor would we model dynamic and static processes within the same equation. The ARFIMA-MLM technique allows us to capitalize on the RCS structure of our dataset while considering the issues unique to time-series data by filtering out temporal and serial autocorrelation using ARFIMA models.

As applied to this dataset, this technique proceeds in five parts. First, we create annual means for the country-level data for each of the dependent $\left(\mathrm{Y}_{\mathrm{t}}\right)$ and independent variables $\left(\mathrm{X}_{\mathrm{t}}\right)$ (Lebo and Weber 2015). Second, we discover the proper noise levels for these dependent and independent variables, as well as, for the units of time $\left(\mathrm{Z}_{\mathrm{t}}\right)$ that do not vary across years (Lebo and Weber 2015). Third, we filter each (X, Y and Z) through its noise model to create units of time series that are free of autocorrelation (Lebo and Weber 2015). Fourth, we remove yearly deterministic components from our country-level data (Lebo and Weber 2015). Finally, we estimate our MLM "in two levels using the double-filtered data" (Lebo and Weber 2015, 246).

This estimation technique (ARFIMA-MLM) provides at least two additional advantages. First, it fixes problems of serial autocorrelation at the country-level by taking deviations across individual observations from units of time (Lebo and Weber 2015, 246). Second, this technique allows us to include additional time unit variables that do not vary within time-points as covariates (Lebo and Weber 2015, 246). Similar to traditional fixed-effects models, ARFIMAMLMs also provide fixed-effects coefficients that represent (in our case) within nation variance. ${ }^{10}$

\section{Results}

When we combine data for all countries with sufficient information we find that for an average country in our data a one degree (Celsius) temperature increase is related to a statistically significant average increase in homicides of nearly $6 \%$ (see Table 1, for coefficients for all variables and Table 2 for specific effect sizes for temperature only). Our control variables show that both male youth unemployment and the consumer price index are positively associated with increases in homicides (with CPI being statistically significant), suggesting that economic factors remain important predictors of homicides. Infant mortality and war casualties, while positively related were not significant. Finally, the proportion of migrants in a country shows a nonsignificant negative correlation with homicides. The results for all countries in our sample thus indicate that climate change is likely to increase the number of homicides worldwide after controlling for several important factors previously identified in the literature. We employed several alternative model specifications and performed robustness checks and found that results held. ${ }^{11}$ It is particularly noteworthy that removing African countries from the analysis does not substantively change our conclusion (the predicted effect decreases marginally from nearly 6 to nearly $5 \%$, but remains highly significant).

-Tables1 and 2 about here- 
Finding a significant positive association between temperatures and homicide levels is consistent with prior research (Anderson Bushman and Groom 1997; Hsiang, Burke and Miguel 2013; Ranson 2014; Rotton and Cohn 2003). Most studies, however, report smaller effects. We suspect that the geographic bias, sample size and modeling techniques of prior work have led to an underestimate of statistical relation between climate change and violence. Given that a few studies indicate that geographic areas may modify the link between temperature and violence we conducted our analysis also on regional levels. Here, we find some peculiar results. At the regional level we found no significant climatic effects for former Soviet countries (-.30\% per degree Celsius), statistically insignificant but positive effects for Europe $(+1.82 \%)$, Asia $(+1.82 \%)$ and Latin America (+4.39\%), a significant effect for North America, and Australasia $(+2.85 \%)$ and a rather strong relation in Africa $(+17.94 \%)$. We are not entirely surprised that the results for former Soviet countries show no connection to rising temperatures. Murders in these countries have been driven by growing alcohol consumption and the growth of organized crime after the dissolution of the Soviet Union (World Health Organization 2006). These two factors mean that many homicides are probably not as dependent on weather conditions as nations with a higher proportion of street gang killings.

Our control variables show some variability across regions, but some general patterns emerge. The two most interesting results are found in variables "Price Index" and "Migrants". For consumer price index the results indicate good support for a positive association, indicating that large annual jumps in prices of products may be linked to increases in homicides. Price increase in consumer goods may trigger a rise in violence in several ways but most likely indicates increases of financial strains of vulnerable populations (Pratt and Godsey 2003). Changes in youth unemployment also are generally positively associated with increases in homicides. This is not entirely unsurprising; as young people fail to make inroads in the labor market alternative avenues are often explored, including associations with street gangs, for instance (Wilson 1987; Anderson 1999; Venkatesh 2006). Such associations are likely to increase a young person's changes to become the victim or offender in a homicide incident (Decker and van Winkle 1996).

Our results favor a negative association between migration and homicide rates. Given that international migration is typically undertaken to seek out educational and labor opportunities it should not be surprising that the proportion of immigrants is negatively associated with homicides in some regions (Stowell, Messner and McGeever 2009; Wadsworth 2010).

While our results indicate a significant connection between climate change and homicide, there are large regional differences in the magnitude of this effect ranging from no discernable connections (former Soviet countries) to insignificant positive effects (Latin America, Europe, Asia), to significant positive correlations (Africa, North America/Australia/New Zealand). We suspect that the reasons for this variability may be highly context dependent. The regions that show greatest support for the link between climate change and homicide are characterized by higher proportions of street violence, perhaps indicating that countries with higher levels of street violence are more likely to experience increasing levels of violence as temperatures increase.

We suspect that the prevalence of certain types of homicides - such as a higher proportion of outdoor killings - are not simply driven by geography and prevailing climates, but are also related to structural social and economic inequalities. Ouiemet (2012) suggests that countries with higher homicides levels are also likely to experience a greater degree of income 
inequality. We believe that countries with higher levels of social and economic inequities produce the conditions (unemployment, educational failure) which promote outdoor activities among at-risk groups (young disenfranchised males). Obviously we cannot be certain about such an explanation since we are not able to test this directly in our data. We encourage researchers to examine disaggregated homicide data from various sites and examine their variability in greater detail.

We tested all of the models in Table 1 under many alternative specifications and conditions to verify the robustness of the findings. To begin, we employed both a traditional fixed-effects and multilevel analysis; results did not substantively change. Next we used our reported model and removed one year at a time and found that results remain largely unchanged. We also removed one country at a time and again discovered little change in the results. Finally, we removed one control variable at a time and the results remain substantially unchanged. ${ }^{12,13}$

\section{Limitations}

Our study uses annual temperatures as a proxy to measure climate change, but temperature is but one element in a changing climate. Our study did not examine seasonal factors, precipitation or extreme weather events. While previous research acknowledges the importance of incorporating other measures of climate change, incorporating them in the current study proved problematic. Precipitation changes, for instance, may impact rates of violence but it makes a theoretical difference if the precipitation comes in short heavy downpours or in protracted event. Using aggregated annual data makes it impossible to discern the type of precipitation events experienced by our sample countries. Similarly, aggregated data make it difficult to discern if very hot summers are responsible for increases in homicides as the homicides may have increased evenly throughout the year or may have even spiked during winter months. Unfortunately the resolution of cross-national homicide data is limited to annual records. Substantial primary data collection would be needed to address this issue.

Although homicide is probably the best cross-national and longitudinal measure of violence, minor differences across contexts exist (Mares 2009). For instance, what is considered legal self-defense in the United States may be considered a homicide in the Netherlands. We do not believe that such definitional and reporting differences would account for more than a small percentage of differences across countries. For our study, a more important issue is sample bias. Although our study is currently the broadest sample available, it oversamples developed countries. This is largely because developed countries have better data recording practices. Given the resulting model differences we can observe between more developed and developing countries we believe the predicted overall climate change effects on violence may be higher if we had been able to collect a more complete sample. For instance, if one excludes African countries from the analysis the correlation coefficient for temperature changes from .056 to .048 (but remains significant). Relatedly, it is also important to remember that the unit of analysis in our study is the country, meaning that our estimated 'climate change effect' on violence is not a 'global' average, but rather the averaged country-specific impact. An average $5.9 \%$ increase in homicides in the countries in our study may mean that India's increase may be only $1.9 \%$, but Ireland's increase may be $9.9 \%$. Given the large population differences between the two countries one quickly realizes that it is impossible from this study to reliably estimate the likely additional homicides generated by climate change. 
The results of this study could likely be improved by examining homicide and climatic data at the city level. Unfortunately such data are rather limited at present and would require substantial data collection efforts. Another issue is that we are unable to directly test existing theories that explain the link between warmer temperatures and violence. It would be important to understand if warmer temperatures directly (GAAM) or indirectly influence violence (RAT). Again, the problem lies in the level of aggregation of the data. For instance, we cannot say if the link discovered is the result of warmer winter temperatures or warmer summer temperatures (or both). Also, since we have no information on the type of homicide incidents and locations in which they occurred it is not an ideal data set to test specific theoretical aspects of the heatviolence link. For this, we encourage researchers to employ data at lower levels of aggregation.

We also cannot offer any direct explanations for the differences in regional models. These differences are no doubt very context dependent and would have to be explored with smaller scale data. We suspect that further disaggregation of homicides may prove fruitful as it may uncover whether regional differences persist once similar types of homicides are compared.

\section{Conclusion}

Although our study confirms the results of several recent studies by finding largely positive relationships between climate change and interpersonal violence, our study did so using a larger sample of countries. Results indicate that 'globally' an average annual increase of one degree Celsius is related to a $5.9 \%$ average increase in national homicide rates. Our results further indicate that the strength and significance of this relationship varies substantially from region to region. Some regions show no significant link between warming and homicides (especially former Soviet countries), others show quite a large connection (particularly African countries). We suspect that the regional variation may rests -in part- on prevailing types of homicides in each region. Prior research, indicates that certain types of homicides are less influenced by a changing climate. For instance, homicides in street settings are more likely influenced by climatic variations, while homicides in a domestic context (and typically occur indoors) are less likely impacted by variation in climatic conditions. Nonetheless, since no contextual indicators are currently available for cross-national homicide data, we cannot be certain that our idea is a valid explanation for the regional variation.

Unfortunately our study unveils, like many others examining the societal impacts of climate change, that those countries and people who have contributed less to current levels of atmospheric greenhouse gasses, are predicted to experience the most negative impacts. We certainly encourage more research on this topic. As the evidence accumulates that climate change is likely to contribute to higher rates of interpersonal violence, many new questions emerge that cannot be adequately answered by national and international level data, but will require the use of high resolution local studies.

\footnotetext{
${ }^{1}$ The authors would like to thank the supportive criticism of the anonymous reviewers.

${ }^{2}$ Please refer to the supplementary materials for more details on the data and analysis.
} 
${ }^{3}$ Many smaller countries with infrequent homicides and a small population were excluded. Please see the Appendix for more details.

${ }^{4}$ The Appendix outlines the practical steps we took to select sites and temperature readings.

${ }^{5}$ Baseline models with temperature as the only independent variable are available in the Appendix.

${ }^{6}$ The Appendix furnishes additional information on all variables, including a correlation matrix between the dependent variable and all independent variables. The Appendix also includes several independent variables that we did not use in the models reported here. Moreover, we provide a selection of models that include at least some of these independent variables in the Appendix.

${ }^{7}$ Countries included in our sample: Algeria, Australia, Austria, Bahamas, Bangladesh, Barbados, Belgium, Belize, Canada, Colombia, Costa Rica, Czech Republic, Egypt, El Salvador, Estonia, Finland, France, Germany, Greece, Honduras, Hungary, India, Ireland, Italy, Jamaica, Japan, Kazakhstan, Kenya, Lithuania, Macedonia, Mexico, Moldova, Mongolia, Morocco, Netherlands, New Zealand, Norway, Pakistan, Paraguay, Philippines, Poland, Portugal, Romania, Serbia, Singapore, South Africa, Spain, Sri Lanka, Sweden, Switzerland, Tajikistan, Thailand, Uganda, United Kingdom, Uruguay, United States, and Venezuela.

${ }^{8}$ We estimated a fixed effects models using the same independent variables as those contained in Table One. The results are substantially similar to those that are reported here, and are contained in the Appendix.

${ }^{9}$ We estimated a multilevel model, without using the ARFIMA technique using the same independent variables as those contained in Table 1 . The results are substantially similar to those that are reported here, and are contained in the Appendix.

${ }^{10}$ For more precise calculations of unit and standard deviation change effects, please refer to the supplementary materials.

${ }^{11}$ The supplemental materials provide more information about and the specific results of the models referenced in this paragraph.

${ }^{12}$ Some of these results are modeled in the Appendix (i.e., country/year fixed effects and removing one control variable at a time), or are contained in the supplemental materials.

${ }^{13}$ These same robustness checks were run on the fixed effects and multilevel modeling (without ARFIMA) techniques that are referenced in earlier parts of this paper. The results are strikingly similar to those that are reported here. 